\title{
Cross-cultural adaptation and validation of the Turkish Yellow Flag Questionnaire in patients with chronic musculoskeletal pain
}

\author{
Meltem Koç ${ }^{1}$, Zilan Bazancir ${ }^{2}$, Hakan Apaydın ${ }^{3}$, Burcu Talu ${ }^{4}$, and Kılıçhan Bayar ${ }^{1}$ \\ 'Department of Physiotherapy and Rehabilitation, Faculty of Health Sciences, Muğla Sitkı Koçman University, Muğla, Turkey \\ ${ }^{2}$ Department of Physiotherapy and Rehabilitation, Faculty of Physical Therapy and Rehabilitation, Hacettepe University, Ankara, Turkey \\ ${ }^{3}$ Department of Rheumatology, Ankara City Hospital, Ankara, Turkey \\ ${ }^{4}$ Department of Physiotherapy and Rehabilitation, Faculty of Health Sciences, Inonu University, Malatya, Turkey
}

Received April 26, 2021

Revised June 29, 2021

Accepted July 23, 2021

Handling Editor: Jee Youn Moon

\section{Correspondence}

Meltem Koç

Department of Physiotherapy and

Rehabilitation, Faculty of Health

Sciences, Muğla Sıtkı Koçman University,

Kötekli street, Muğla 48000, Turkey

Tel: +90-507-3697866

Fax: $+90-252-2111880$

E-mail: kcmeltemm@gmail.com
Background: Yellow flags are psychosocial factors shown to be indicative of longterm chronicity and disability. The purpose of the study was to evaluate the psychometric properties of the Turkish Yellow Flag Questionnaire (YFQ) in patients with chronic musculoskeletal pain (CMP).

Methods: The cross-cultural adaptation was conducted with translation and backtranslation of the original version. Reliability (internal consistency and test-retest) was examined for 231 patients with CMP. Construct validity was assessed by correlating the YFQ with the Hospital Anxiety and Depression Scale (HADS), Orebro Musculoskeletal Pain Questionnaire (OMPQ), and Tampa Kinesiophobia Scale (TKS). Factorial validity was examined with both exploratory and confirmatory factorial analysis.

Results: The YFQ showed excellent test/retest reliability with an Intraclass correlation coefficient of 0.82 . The internal consistency was moderate (Cronbach's alpha of 0.797 ). As a result of the exploratory factor analysis, there were 7 domains compatible with the original version. As a result of confirmatory factor analysis, the seven-factor structure of YFQ was confirmed. There was a statistically significant correlation between YFQ-total score and OMPQ $(r=0.57, P<0.001)$, HADS-anxiety $(r$ $=0.32, P<0.001)$, HADS-depression $(r=0.44, P<0.001)$, and TKS $(r=0.37, P<$ 0.001).

Conclusions: This study's results provide considerable evidence that the Turkish version of the YFQ has appropriate psychometric properties, including test-retest reliability, internal consistency, construct validity and factorial validity. It can be used for evaluating psychosocial impact in patients with CMP.

Key Words: Anxiety; Chronic Pain; Cross-Cultural Comparison; Depression; Factor Analysis, Statistical; Musculoskeletal Pain; Patient Reported Outcome Measures; Psychometrics; Reproducibility of Results; Surveys and Questionnaires; Validation Study.

\section{INTRODUCTION}

Yellow flags are the name given to a group of psychosocial risk factors (e.g., depression, psychological distress, passive coping strategies, and fear-avoidance beliefs) that explain long term chronicity and disability in chronic pain. (a) This is an open-access article distributed under the terms of the Creative Commons Attribution Non-Commercial License (http://creativecommons.org/licenses/by-nc/4.0/), which permits unrestricted non-commercial use, distribution, and reproduction in any medium, provided the original work is properly cited.

(c) The Korean Pain Society, 2021
Author contributions: Meltem Koç: Writing/manuscript preparation; Zilan Bazancir: Investigation; Hakan Apaydın: Investigation; Burcu Talu: Supervision; Kılıçhan Bayar: Methodology. 
Therefore, yellow flags are predicters of nonreturn to work, transformation from acute to chronic, and disability [1]. It is known that the most important approach in preventing the transition to chronic pain, especially in acute low back pain, is the early detection of psychosocial risk factors [2].

If there are no signs that could lead to an emergency medical attention (tumors, fractures, etc.), called "red flags", the doctor or therapist should consider yellow flags. Evaluation of yellow flags must be evidence-based, which means a high standardization, adequate reliability, and verification with prospective studies [3]. The use of crossculturally adapted self-reported outcomes in different languages facilitates the collection of reliable data in studies that are conducted in different countries. It also allows for comparison of the results.

The Yellow Flag Questionnaire (YFQ) is a recently developed, multidimensional assessment tool used for assessment of pain-related psychosocial risk factors among individuals with chronic musculoskeletal pain (CMP) [4]. The tool provides a comprehensive assessment of psychosocial risk factors related to chronic pain in 32 items and 7 subgroups (activity, work, emotions, harm \& blame, diagnosis beliefs, co-morbidity, and control). The most important difference from other small number of multi-dimensional yellow flag assessments tools is that it can conduct more comprehensive risk factor research [5-7]. The YFQ was developed in English and there is clearly a need for a tool that enables the assessment of psychosocial risk factors in chronic pain in a single tool in the Turkish community. Therefore, this study aimed to adapt the YFQ cross-culturally for use in Turkish speaking patients and to assess the reliability and validity of the translated version.

\section{MATERIALS AND METHODS}

\section{Participants}

Firstly, the necessary permission was obtained by e-mail from the corresponding author of the original article. Then, application was made to the Health Sciences Ethics Committee of İnönü University to carry out the study, and the necessary permission was obtained (2018/7-10).

Guidelines for the respondent-to-item ratio range from 5:1 (i.e., fifty respondents for a 10-item questionnaire), to $10: 1,15: 1$ or $30: 1$ [8]. For this reason, it was the goal to have a minimum of 160 participants for the 32 -item YFQ. Finally, the study was completed with 231 participants. The study was conducted in the Physical Therapy and Rehabilitation department outpatient clinic of a university hospital between July 2018 and December 2019.

To be included, the following criteria were determined:
(1) age between 18 and 65 years, (2) having had CMP for at least 3 months, and (3) native Turkish speakers and were to be at least literate. Patients with any psychological (i.e., schizophrenia, delirium, acute confusional disorders) and cognitive disorder, and having had "red flags" signs (e.g., cauda equina syndrome, tumor, inflammatory diseases, or other symptoms that required urgent intervention) were excluded. The aim of the study, the methods, and the expected results were explained to the participants, and a signed informed consent form was obtained.

\section{Cross-cultural adaptation}

Forward-backwards translation method was used for the translation process. It is the most used translation method [9]. YFQ was translated into Turkish by two different and independent translators whose first language was Turkish (non-professional native Turkish speaker). Inconsistencies between the two translations were resolved by the joint decision of the translators and a physiotherapist. Then the back translation method was applied. Two different and independent translators whose native language is English and who do not have a medical background translated the Turkish questionnaire back into English. Finally, all translators and research team met for inconsistencies between this English version of the questionnaire and its original version. The penultimate Turkish version of the YFQ was then created.

Content validity was investigated before starting the validity and reliability studies of the Turkish YFQ. The opinions of 10 physiotherapy specialists were obtained for content validity and a pilot application was performed with 15 Turkish speaking chronic pain patients. There was no response from the experts and patients about any change. The final version of the Turkish YFQ was completed for further testing.

\section{Reliability}

Reliability is a term related to the extent to which results can be reproduced when research is repeated under the same conditions. In this study, internal consistency (homogeneity) and test-retest reliability (reproducibility) were investigated for reliability.

In total, 231 patients were asked to complete all outcome measures. Sixty of them completed the YFQ twice, with an interval of seven days, to assess its test-retest reliability. All assessments were performed face-to-face in the clinic.

\section{Validity}

Validity is a term that relates to the extent to which results 
actually measure what they are supposed to measure. In this study, the structure and factorial validity were investigated.

Construct validity is a concept related to how much a survey is related to other instruments that measure the same structure. In this study, the Hospital Anxiety and Depression Scale (HADS) [10], Orebro Musculoskeletal Pain Questionnaire (OMPQ) [11], and Tampa Kinesiophobia Scale (TKS) [12] were used while investigating the construct validity of the YFQ. The reason for choosing the HADS and TKS questionnaires is that the YFQ contains items investigating kinesophobia, depression and anxiety. The reason for choosing the OMPQ questionnaire is that it investigates psychosocial risk factors in CMP patients. Therefore, the OMPQ is the gold standard comparison tool for the YFQ.

The factorial validity is used to determine the subgroups and homogeneity of the questionnaire. For this, exploratory factor analysis and confirmatory factor analysis were used in statistical analysis.

\section{Instruments}

1) YFQ

The original language of the YFQ is in English, and it is a patient-based self-report questionnaire developed by Cornelia Rolli Salathé, evaluating psychosocial impact in patients with CMP. The questionnaire consists of 32 items and 7 domains (activity, co-morbidity, diagnosis beliefs, emotions, harm \& blame, pain control, and work factors). Each item is scored between $0-4$, however 7 items (items $1,4,5,6,14,26$, and 30) are reverse scored. The total score ranges from 0-100, and high scores indicate that individuals' multi-factor psychosocial states are affected due to chronic pain. The original version of the YFQ was found to have high reliability (Cronbach alpha $=0.91$ ) and discriminant validity [4].

\section{2) OMPQ}

The OMPQ is a 25-item tool that enables the assessment of psychosocial risk factors, which are yellow flags, in individuals with musculoskeletal pain. Twenty-one of the 25 items are scored on a scale from 0 to 10 . The scored-items assess work absence due to pain, pain duration, pain location, pain intensity, control over pain, frequency of pain episodes in the past 3 months, functional ability, mood, fear-avoidance, and perceptions of work. The scored items are summed to provide a total score, ranging between 0 and 210, with higher scores indicating a higher risk of a poor outcome [13]. The Turkish version of the OMPQ was created by Öncü et al. [11].

\section{3) HADS}

HADS is a 14-item assessment tool for anxiety and depression in non-psychiatric patients. Seven of the items are about anxiety and seven relate to depression. Each item is scored between $0-3$. Total scores range from 0 to 21 for both sets of measurements, with higher scores indicating greater anxiety and depression [14]. Turkish validity and reliability have been demonstrated by Aydemir et al. [10]

\section{4) TKS}

TKS is a 17-items assessment tool for kinesiophobia in chronic pain. Each item has a 4-point Likert scale and the total score varies between 17 and 68. A total score is calculated after inversion of the individual scores of items 4, 8, 12 , and 16. A high value on the TKS indicates a high degree of kinesiophobia [15]. Turkish validity and reliability have been demonstrated by Yilmaz et al. [12].

\section{Statistical analysis}

Statistical analysis was performed using the SPSS program for Windows, version 22.0 (IBM Co., Armonk, NY). Confirmatory factor analysis was performed with the SPSS AMOS 21 program. Descriptive data for the participants' characteristics (e.g., age, sex, pain duration, and education status, among others) and outcome measures were expressed as mean \pm standard deviation (SD), median, or percentage (\%).

The internal consistency for the Turkish version of the YFQ was assessed using Cronbach's alpha. A Cronbach's result of at least 0.80 was considered good consistency, of between 0.80 and 0.70 was considered moderate, and of less than 0.70 was considered low [16]. Test-retest reliability was assessed by intraclass correlation coefficients (ICC). The ICC value is between 0.00 and 1.00, values between $0.60-0.80$ indicate good reliability, and values greater than 0.80 indicate excellent reliability.

Construct validity was analyzed using the Spearman correlation coefficient. This coefficient ranges from -1 to +1 [16]. The level of significance was determined at $P<0.05$. A summary of the statistical analysis performed is given in Table 1.

\section{RESULTS}

A total of 231 patients with CMP (127 female, 104 male) participated in this study. Most patients complained of 
pain in the low back (46\%) and neck region (29\%). The average pain duration was $93.1 \pm 102.7$ months. Almost half of the patients used a drug once a week because of pain (52\%). Demographic and clinical variables of patients are presented in Table 2.

The YFQ total score was found to be $63.32 \pm 16.75$, the OMPQ total score was $98.59 \pm 32.29$, the HADS-anxiety total score was $7.79 \pm 3.47$, the HADS-depression total score was $6.84 \pm 3.11$, and the TKS total score was $33.83 \pm 8.64$. Total scores of the questionnaires are given in Table 3.

\section{Reliability}

Data from all 231 participants were analyzed to assess reliability. The internal consistency of the Turkish version of the YFQ, measured by the Cronbach's alpha coefficient, was moderate (alpha $=0.797)$. The ICC coefficients for test-retest reliability were 0.762 (95\% confidence interval:

Table 1. A summary of the statistical analysis performed

\begin{tabular}{ll}
\hline Language validity & Forward-backward translation method \\
Content validity & Expert opinion \\
Cultural adaptation & Pre-test group \\
Factorial validity & Explanatory and confirmatory factor analysis \\
Construct validity & Correlation analysis with other instruments \\
Reliability & Test-retest analysis \\
& Cronbach's $\alpha$ reliability analysis \\
\hline
\end{tabular}

0.713-0.804). All items showed good reliability.

\section{Validity}

The construct validity of the YFQ was measured by the Spearman correlation coefficient between the YFQ and the OMPQ, HADS, and TKS. There was a statistically significant correlations between the YFQ (total score) and the OMPQ ( $\mathrm{r}=0.57, P<0.001)$, HADS-anxiety ( $\mathrm{r}=0.32, P<$ o.ool), HADS-depression ( $\mathrm{r}=0.44, P<0.001)$, and TKS ( $\mathrm{r}=$ $0.37, P<0.001)$. Correlation results between the YFQ and other instruments are given in detail in Table 4.

The solution that emerged from the factor analysis revealed seven factors with eigenvalues of $7.10,4.96,3.58$, $3.24,2.96,2.31$, and 1.99 which accounted for $81.77 \%$ of the observed variance. The factor loads of the items varied between 0.363 and 0.846 . The factors that were extracted in this study were consistent with all the domains (activity, work, emotions, harm \& blame, diagnosis beliefs, comorbidity, and control) of the original version of the YFQ. The factor loadings of all items are presented in Table 5.

In order to test the seven-domain model structure of the YFQ, confirmatory factor analysis was performed. According to the confirmatory factor analysis, it was determined that the structural equation model result of the YFQ was significant at the $P=0.000$ level, and that the 32 items and 7 domains constituting the scale were related to the scale structure. As a result of confirmatory factor analysis, the

Table 2. Demographic characteristics of patients $(n=231)$

\begin{tabular}{|c|c|c|}
\hline & Variable & Value \\
\hline Age (yr) & & $36.7 \pm 8.4(25-59)$ \\
\hline Pain duration (mo) & & $93.1 \pm 102.7(3-528)$ \\
\hline \multirow[t]{2}{*}{ Sex } & Female & $127(55.0)$ \\
\hline & Male & $104(45.0)$ \\
\hline \multirow[t]{5}{*}{ Education status } & Literate & $14(6.1)$ \\
\hline & Primary school & $36(15.6)$ \\
\hline & High school & $72(31.2)$ \\
\hline & University & $77(33.3)$ \\
\hline & Postgraduate & 32 (13.9) \\
\hline \multirow[t]{2}{*}{ Material status } & Married & $164(71.0)$ \\
\hline & Single & $67(29.0)$ \\
\hline \multirow[t]{3}{*}{ Work status } & Not working & 75 (32.5) \\
\hline & Working & $135(58.4)$ \\
\hline & Student & $21(9.1)$ \\
\hline \multirow[t]{3}{*}{ Use of opioids } & None & 77 (33.3) \\
\hline & Once a day or week & $122(52.8)$ \\
\hline & Once a month & $32(13.9)$ \\
\hline \multirow[t]{4}{*}{ Pain anatomic region } & Low back (chronic back/spine pain, spondyloarthropathy, post-surgical back problems) & $108(46.8)$ \\
\hline & Neck (chronic neck pain) & $67(29.0)$ \\
\hline & Upper extremity (chronic shoulder pain, tendinopathy, carpal tunnel syndrome) & $24(10.4)$ \\
\hline & Lower extremity (joint/muscle myofascial pain, meniscal problems) & $32(13.9)$ \\
\hline
\end{tabular}

Values are presented as mean \pm standard deviation (minimum-maximum) or number (\%). 
Table 3. Characteristics of outcome measures

\begin{tabular}{|c|c|c|c|c|c|}
\hline Questionnaire & Item number & Response & Total score & Better function indicated by & Mean \pm standard deviation \\
\hline YFQ-total & 32 & $0-4$ & $0-128$ & Low score & $63.32 \pm 16.75$ \\
\hline YFQ-activity & & & $0-28$ & & $13.90 \pm 4.10$ \\
\hline YFQ-work & & & $0-16$ & & $6.67 \pm 4.25$ \\
\hline YFQ-emotion & & & $0-24$ & & $12.38 \pm 3.62$ \\
\hline YFQ-harm_blame & & & $0-16$ & & $6.54 \pm 4.44$ \\
\hline YFQ-belief & & & $0-8$ & & $5.61 \pm 2.96$ \\
\hline YFQ-comorbidity & & & $0-8$ & & $3.41 \pm 2.65$ \\
\hline YFQ-control & & & $0-28$ & & $14.78 \pm 5.03$ \\
\hline OMPQ & 25 & $0-10$ & $0-210$ & Low score & $98.59 \pm 32.29$ \\
\hline HADS-anxiety & 14 & $0-3$ & $0-21$ & Low score & $7.79 \pm 3.47$ \\
\hline HADS-depression & & & $0-21$ & & $6.84 \pm 3.11$ \\
\hline TKS & 17 & $1-4$ & $17-68$ & Low score & $33.83 \pm 8.64$ \\
\hline
\end{tabular}

YFQ: Yellow Flag Questionnaire, OMPQ: Orebro Musculoskeletal Pain Questionnaire, HADS: Hospital Anxiety and Depression Scale, TKS: Tampa Kinesiophobia Scale.

Table 4. Correlation coefficient ( $r$ ) between YFQ and OMPQ, HADS, TKS

\begin{tabular}{|c|c|c|c|c|}
\hline YFQ & $\begin{array}{c}\text { OMPQ } \\
\text { r }(95 \% \mathrm{Cl})\end{array}$ & $\begin{array}{l}\text { HADS-anxiety } \\
\text { r (95\% Cl) }\end{array}$ & $\begin{array}{c}\text { HADS-depression } \\
\text { r (95\% Cl) }\end{array}$ & $\begin{array}{c}\text { TKS } \\
r(95 \% \mathrm{Cl})\end{array}$ \\
\hline YFQ_total & $0.575 * *(0.48$ to 0.70$)$ & $0.321 * *(0.22$ to 0.41$)$ & $0.440 * *(0.34$ to 0.55$)$ & $0.375 * *(0.23$ to 0.47$)$ \\
\hline YFQ_activity & $0.189 * *$ (0.0 to 0.27$)$ & $0.211 * *(0.79$ to 0.33$)$ & $0.105(-0.6$ to 0.14$)$ & $0.473 * *(0.31$ to 0.52$)$ \\
\hline YFQ_work & $0.232 * *(0.16$ to 0.48$)$ & 0.034 (0.10 to -0.16$)$ & $0.202 * *(0.12$ to 0.35$)$ & $0.199 * *$ (0.10 to 0.32$)$ \\
\hline YFQ_emotion & $0.206 * *(0.11$ to 0.33$)$ & $0.237 * *(0.10$ to 0.29$)$ & $0.154 *$ (0.03 to 0.28$)$ & $0.397 * *(0.25$ to 0.46$)$ \\
\hline YFQ_harm_blame & $0.588 * *(0.46$ to 0.64$)$ & $0.172 * *(0.0$ to 0.28$)$ & $0.257 * *$ (0.16 to 0.35$)$ & $0.198 * *(0.10$ to 0.33$)$ \\
\hline YFQ_belief & 0.004 (0.0 to 0.02) & $-0.127(-0.25$ to 0.04$)$ & $0.131 *$ (0.01 to 0.23$)$ & $-0.140 *(-0.26$ to 0.06$)$ \\
\hline YFQ_comorbidity & $0.703 * *(0.64$ to 0.79$)$ & $0.605 * *(0.51$ to 0.65$)$ & $0.704 * *$ (0.65 to 0.77$)$ & $0.523 * *(0.43$ to 0.59$)$ \\
\hline YFQ_control & $0.479 * *(0.40$ to 0.66$)$ & 0.071 (0.02 to 0.12$)$ & $0.514 * *(0.47$ to 0.68$)$ & $0.211 * *(0.12$ to 0.32$)$ \\
\hline
\end{tabular}

YFQ: Yellow Flag Questionnaire, OMPQ: Orebro Musculoskeletal Pain Questionnaire, HADS: Hospital Anxiety and Depression Scale, TKS: Tampa Kinesiophobia Scale, r: Spearman correlation coefficient, Cl: confidence interval. $* P<0.05 . * * P<0.001$.

$\mathrm{X} 2 / \mathrm{df}$ was 1.692; the goodness of fit index value was 0.88 , the comparative fit index value was 0.96 , and the root mean square error of approximation value was 0.076 . Since these values were within acceptable limits, the sevenfactor structure of the YFQ was confirmed.

\section{DISCUSSION}

The YFQ is a guiding outcome measure for a comprehensive assessment of psychosocial risk factors in chronic pain. However, to the best of our knowledge, the YFQ has not been translated into other languages, and the first cross-cultural adaptation was made to Turkish. The Turkish version of the YFQ demonstrated satisfactory reliability and validity in the Turkish CMP population in line with the original version.

The original version was conducted with 254 injured workers who had CMP (mean age $=37.2$ years, $\mathrm{SD}=11.9$, $53.5 \%$ men) [4]. The Turkish version in this study was con- ducted with 231 patients with CMP (mean age $=36.7$ years, $\mathrm{SD}=8.4,45 \% \mathrm{men}$ ). It is stated that there should be at least 30 individuals, in the literature, for test-retest reliability analysis [17]. The test-retest reliability of the Turkish version of the YFQ was evaluated with 60 patients. The result was moderate, with a correlation coefficient of 0.82 . The internal consistency of the Turkish version of the YFQ was moderate, with a Cronbach's alpha coefficient of 0.79 . The original version of the YFQ also had excellent internal consistency, with a Cronbach's alpha coefficient of 0.91 . The literature states that the Cronbach's alpha coefficient should be higher than 0.70 [18].

After the exploratory factor analysis in this study, seven factors accounted for $81.77 \%$ of the observed variance, and the factor loads of the items varied between 0.363 and 0.846 for the Turkish version of the YFQ. It is stated in the literature that it is enough to have factor loads of 0.30 and above, and variance rates between $40 \%-60 \%$ [19]. Therefore, no items were discarded. Only confirmatory factor analysis was used to determine the factor structure in the original 
Table 5. Forced seven-factor solution by principal axis factoring of items from the YFQ $(n=231)$

\begin{tabular}{|c|c|c|c|c|c|c|}
\hline \multirow{2}{*}{ Component } & \multicolumn{3}{|c|}{ Initial eigenvalues } & \multicolumn{3}{|c|}{ Extraction sums of squared loadings } \\
\hline & Total & $\%$ of variance & Cumulative \% & Total & $\%$ of variance & Cumulative \% \\
\hline 1 & 7.107 & 22.209 & 22.209 & 7.107 & 22.209 & 22.209 \\
\hline 2 & 4.960 & 15.500 & 37.708 & 4.960 & 15.500 & 37.708 \\
\hline 3 & 3.580 & 11.188 & 48.897 & 3.580 & 11.188 & 48.897 \\
\hline 4 & 3.246 & 10.142 & 59.039 & 3.246 & 10.142 & 59.039 \\
\hline 5 & 2.965 & 9.266 & 68.305 & 2.965 & 9.266 & 68.305 \\
\hline 6 & 2.317 & 7.240 & 75.546 & 2.317 & 7.240 & 75.546 \\
\hline 7 & 1.994 & 6.230 & 81.775 & 1.994 & 6.230 & 81.775 \\
\hline 8 & 1.382 & 4.318 & 86.093 & & & \\
\hline 9 & 1.252 & 3.913 & 90.006 & & & \\
\hline 10 & 1.039 & 3.246 & 93.252 & & & \\
\hline 11 & 0.673 & 2.103 & 95.355 & & & \\
\hline 12 & 0.541 & 1.689 & 97.044 & & & \\
\hline 13 & 0.369 & 1.152 & 98.196 & & & \\
\hline 14 & 0.277 & 0.865 & 99.061 & & & \\
\hline 15 & 0.173 & 0.540 & 99.601 & & & \\
\hline 16 & 0.128 & 0.399 & 100.000 & & & \\
\hline 17 & - & - & 100.000 & & & \\
\hline 18 & - & - & 100.000 & & & \\
\hline 19 & - & - & 100.000 & & & \\
\hline 20 & - & - & 100.000 & & & \\
\hline 21 & - & - & 100.000 & & & \\
\hline 22 & - & - & 100.000 & & & \\
\hline 23 & - & - & 100.000 & & & \\
\hline 24 & - & - & 100.000 & & & \\
\hline 25 & - & - & 100.000 & & & \\
\hline 26 & - & - & 100.000 & & & \\
\hline 27 & - & - & 100.000 & & & \\
\hline 28 & - & - & 100.000 & & & \\
\hline 29 & - & - & 100.000 & & & \\
\hline 30 & - & - & 100.000 & & & \\
\hline 31 & - & - & 100.000 & & & \\
\hline 32 & - & - & 100.000 & & & \\
\hline
\end{tabular}

Extraction method: principal component analysis.

YFQ: Yellow Flag Questionnaire.

study.

While construct validity was investigated with the HADS, Neck Failure Index, and Spinal Function Ranking (for self-efficacy) in the original study [4], the TKS and OMPQ were used together with the HADS, considering it to be more appropriate in this study. In our results, the YFQ showed a moderate correlation with the OMPQ and TKS. In the original study, there was a 0.66 and 0.73 correlation coefficient between the YFQ-total score and the HADSanxiety and HADS-depression scores, whereas these were 0.32 and 0.44 in this study, respectively. The correlation coefficients related with anxiety and depression were not similar to that of the original study. Although the original study was carried out with injured workers, this study did not contain restrictions on work. It may show higher depression and anxiety scores and better correlation with the YFQ as individuals may have anxiety about returning to work due to injury. More research is needed to better understand this result or inference.

The current study has some strengths. One strength is that the YFQ is the only multidimensional screening scale in the literature that provides a comprehensive assessment of psychosocial risk factors that can be recovery obstacles in patients with CMP. In this sense, it will be able to meet the needs of researchers and clinicians working in this field. Another strength is that this study is the first to investigate the relationship between the YFQ and the OMPQ and TKS. A significant correlation result of the YFQ with the TKS and OMPQ was expected. We think that kinesiophobia may be one of yellow flags in patients with CMP or fear of movement may interact with yellow flags caused by chronic pain-related psychological distress. The OMPQ, 
on the other hand, is the gold standard benchmarking tool for the YFQ, as mentioned earlier.

The current study also had several limitations. First, while the YFQ aimed for a comprehensive assessment of the yellow flags, this led to the substantial length of the questionnaire. This can make such questionnaires less useful. The second limitation is that the sample in this study consists of patients who received only physical therapy and did not have psychological disorders. However, chronic pain problems accompanied by a psychological disorder are common [20]. This situation prevents the generalizability of the questionnaire to all CMP patients. The third limitation is that some features for the YFQ have not been investigated in this study. In future studies, cut-off value for the YFQ, responsiveness to clinical change, and predictive ability should be investigated.

This study's results provide considerable evidence that the Turkish version of the YFQ has appropriate psychometric properties, including test-retest reliability, internal consistency, construct validity, and factorial validity. The YFQ multidimensional screening scale is recommended for use in assessing psychosocial risk factors in patients with CMP.

\section{CONFLICT OF INTEREST}

No potential conflict of interest relevant to this article was reported.

\section{FUNDING}

No funding to declare.

\section{ORCID}

Meltem Koç, https://orcid.org/0000-0001-6456-8779

Zilan Bazancir, https://orcid.org/0000-0001-6834-8343

Hakan Apaydın, https://orcid.org/0000-0001-7219-1457

Burcu Talu, https://orcid.org/0000-0002-5623-8291

Kılıçhan Bayar, https://orcid.org/0000-0002-8090-5859

\section{REFERENCES}

1. Kendall N. Guide to assessing psychosocial yellow flags in acute low back pain: risk factors for long-term disability and work loss. January 1997 ed. Wellington, Accident Rehabilitation and Compensation Insurance Corporation, The National Health Committee. 1997.
2. Kovacs FM, Abraira V, Zamora J, Fernández C. The transition from acute to subacute and chronic low back pain: a study based on determinants of quality of life and prediction of chronic disability. Spine (Phila Pa 1976) 2005; 30: 1786-92.

3. Main CJ, Kendall NAS, Hasenbring MI. Screening of psychosocial risk factors (yellow flags) for chronic back pain and disability. In: From acute to chronic back pain: risk factors, mechanisms, and clinical implications. Edited by Hasenbring MI, Rusu AC, Turk DC. Oxford, Oxford University Press. 2012.

4. SalathéCR, Trippolini MA, Terribilini LC, Oliveri M, Elfering A. Assessing psycho-social barriers to rehabilitation in injured workers with chronic musculoskeletal pain: development and item properties of the Yellow Flag Questionnaire (YFQ). J Occup Rehabil 2018; 28: 365-76.

5. Mannion AF, Mariaux F, Reitmeir R, Fekete TF, Haschtmann D, Loibl M, et al. Development of the "Core Yellow Flags Index" (CYFI) as a brief instrument for the assessment of key psychological factors in patients undergoing spine surgery. Eur Spine J 2020; 29: 1935-52.

6. Butera KA, George SZ, Lentz TA. Psychometric evaluation of the Optimal Screening for Prediction of Referral and Outcome Yellow Flag (OSPRO-YF) tool: factor structure, reliability, and validity. J Pain 2020; 21: 557-69.

7. Grotle M, Vøllestad NK, Brox JI. Screening for yellow flags in first-time acute low back pain: reliability and validity of a Norwegian version of the Acute Low Back Pain Screening Questionnaire. Clin J Pain 2006; 22: 458-67.

8. Tsang S, Royse CF, Terkawi AS. Guidelines for developing, translating, and validating a questionnaire in perioperative and pain medicine. Saudi J Anaesth 2017; 11(Suppl 1): S80-9.

9. Danielsen AK, Pommergaard HC, Burcharth J, Angenete E, Rosenberg J. Translation of questionnaires measuring health related quality of life is not standardized: a literature based research study. PLoS One 2015; 10: e0127050.

10. Aydemir Ö, Güvenir T, Küey L, Kültür S. [Validity and reliability of Turkish version of Hospital Anxiety and Depression Scale]. Türk Psikiyatr Derg 1997; 8: 280-7. Turkish.

11. ÖncüJ, Ilişer R, Kuran B. Cross-cultural adaptation of the Orebro Musculoskeletal Pain Questionnaire among Turkish workers with low back pain. J Back Musculoskelet Rehabil 2016; 29: 135-43.

12. Tunca Yılmaz Ö, Yakut Y, Uygur F, Uluğ N. [Turkish version of the Tampa Scale for Kinesiophobia and its test-retest reliability]. Fizyoterapi Rehabil 2011; 22: 44-9. Turkish.

13. Linton SJ, Boersma K. Early identification of patients at risk of developing a persistent back problem: the predictive validity of the Örebro Musculoskeletal Pain Questionnaire. Clin J Pain 2003; 19: 80-6.

14. Zigmond AS, Snaith RP. The hospital anxiety and depression scale. Acta Psychiatr Scand 1983; 67: 361-70.

15. Lundberg MK, Styf J, Carlsson SG. A psychometric evaluation 
of the Tampa Scale for Kinesiophobia - from a physiotherapeutic perspective. Physiother Theory Pract 2004; 20: 121-33.

16. Andresen EM. Criteria for assessing the tools of disability outcomes research. Arch Phys Med Rehabil 2000; 81(12 Suppl 2): S15-20.

17. Marx RG, Menezes A, Horovitz L, Jones EC, Warren RF. A comparison of two time intervals for test-retest reliability of health status instruments. J Clin Epidemiol 2003; 56: 730-5.

18. Terwee CB, Bot SD, de Boer MR, van der Windt DA, Knol DL, Dekker J, et al. Quality criteria were proposed for measure- ment properties of health status questionnaires. J Clin Epidemiol 2007; 60: 34-42.

19. Gaskin CJ, Happell B. On exploratory factor analysis: a review of recent evidence, an assessment of current practice, and recommendations for future use. Int J Nurs Stud 2014; 51: 511-21.

20. Lee HJ, Choi EJ, Nahm FS, Yoon IY, Lee PB. Prevalence of unrecognized depression in patients with chronic pain without a history of psychiatric diseases. Korean J Pain 2018; 31: 11624. 